\title{
Vitamin D attenuates hyperoxia-induced lung injury through downregulation of Toll-like receptor 4
}

\author{
LI YAO, YONGYAN SHI, XINYI ZHAO, ANA HOU, YUJIAO XING, JIANHUA FU and XINDONG XUE \\ Department of Pediatrics, Shengjing Hospital of China Medical University, Shenyang, Liaoning 110004, P.R. China
}

Received December 4, 2016; Accepted March 30, 2017

DOI: 10.3892/ijmm.2017.2961

\begin{abstract}
With considerable morbidity and mortality, bronchopulmonary dysplasia (BPD) is a focus of attention in neonatology. Hyperoxia-induced lung injury has long been used as a model of BPD. Among all the signaling pathways involved, Toll-like receptor 4 (TLR4) has been demonstrated to play an important role, and is known to be regulated by vitamin D. This study aimed at elucidating the effect of vitamin $\mathrm{D}$ on hyperoxia-induced lung injury and the role of TLR4 in the process. Vitamin D was administered to hyperoxia-treated neonatal rats to investigate changes in the morphology of lungs and expression of pro-inflammatory cytokines, apoptotic proteins and TLR4. Vitamin D attenuated hyperoxia-induced lung injury by protecting the integrity of the lung structure, decreasing extracellular matrix deposition and inhibiting inflammation. The upregulation of TLR4 by hyperoxia was ameliorated by vitamin D and apoptosis was reduced. Vitamin D administration antagonized the activation of TLR4 and therefore alleviated inflammation, reduced apoptosis and preserved lung structure.
\end{abstract}

\section{Introduction}

Bronchopulmonary dysplasia (BPD) is a severe complication of preterm birth which causes considerable mortality and morbidity in premature infants. Characterized by multifactorial chronic lung disease, it may affect not only pulmonary function, but also growth, cardiovascular health and neurodevelopment (1). Although the mechanism of BPD yet remains to be clarified, chorioamnionitis, sepsis, invasive mechanical ventilation and hyperoxia may contribute to its incidence (2). Modulation of alveolarization, epithelial cell transdifferentiation, fibroblast and myofibroblast differentiation, extracellular matrix production and inflammation are all involved in the pathological process (3).

Correspondence to: Professor Jianhua Fu, Department of Pediatrics, Shengjing Hospital of China Medical University, 36 Sanhao Street, Shenyang, Liaoning 110004, P.R. China E-mail: fujh@sj-hospital.org

Key words: vitamin D, hyperoxia-induced lung injury, Toll-like receptor 4 , apoptosis, bronchopulmonary dysplasia
Among all the pathways, Toll-like receptor 4 (TLR4) has been demonstrated to play an important role. Hyperoxia has been reported to upregulate TLR4 and activate the nuclear factor- $\kappa \mathrm{B}(\mathrm{NF}-\kappa \mathrm{B})$ pathway $(4,5)$. Antagonists of TLR4 were found to relieve apoptosis and inflammatory response in neurological diseases (6). However, the role of TLR4 is not univocal, as other studies have reported a protective role of TLR4 activation (7). In this case, the exact consequence of TLR4 activation warrants further attention.

The anti-inflammatory and anti-apoptotic effects of vitamin $\mathrm{D}$ have been confirmed in many organs including the lung (8-11). Vitamin D-knockout mice experience a more severe inflammatory response than wild-type mice after LPS treatment (12). The regulatory effect of vitamin D and its receptor on TLR4 has been demonstrated in many studies (13-15). Our previous study also found that vitamin D could significantly relieve LPS-induced lung injury (11). Since TLR4 is the key receptor of LPS, we hypothesized that regulation of TLR4 may be associated with this protective effect of vitamin $D$.

In this study, we established a model of hyperoxia-induced lung injury in neonatal rats. Vitamin D treatment was administered to investigate its effect on lung structure, inflammatory response and apoptosis. We aimed at elucidating the effect of vitamin $\mathrm{D}$ on hyperoxia-induced lung injury and the role of TLR4 in the process.

\section{Materials and methods}

Animal preparation and hyperoxia exposure. All animal procedures were reviewed and approved by the Laboratory Animal Ethics Committee of China Medical University. This study was carried out in strict compliance with the approved protocols. Pregnant Wistar rats (200-250 g) were supplied by the Animal Laboratory, Experimental Research Center, Shengjing Hospital, China Medical University. All rats were maintained in specific pathogen-free static cages with a 12-h light/dark cycle. Chow pellets and tap water were available ad libitum. Hyperoxia exposure was administered as previously described (16). The oxygen concentration was continuously monitored using a strip-chart recorder (model 572; Servomex, Norwood, MA, USA).

Vitamin D treatment. The hyperoxia and normoxia groups were randomly divided into two subgroups. One subgroup was treated with a vitamin D (VD) analogue, paricalcitol 
Table I. Primer sequences used for real-time PCR.

\begin{tabular}{lll}
\hline Primer name & \multicolumn{1}{c}{ Forward $\left(5^{\prime}-3 '\right)$} & Reverse $\left(3^{\prime}-5{ }^{\prime}\right)$ \\
\hline Rat TNF- $\alpha$ & ATGTGGAACTGGCAGAGGAG & TGGAACTGATGAGAGGGAGC \\
Rat IL-1 $\beta$ & ACTCATTGTGGCTGTGGAGA & TAGCAGGTCGTCATCATCCC \\
Rat IL-6 & CCACTGCCTTCCCTACTTCA & TTCTGACAGTGCATCATCGC \\
Rat MIP-2 & AACATCCAGAGCTTGACGGT & ACGATCCTCTGAACCAAGGG \\
Rat IFN- $\gamma$ & GTGTCATCGAATCGCACCTG & GGTGACAGCTGGTGAATCAC \\
Rat $\mathrm{MCP}-1$ & GCTGCTACTCATTCACTGGC & ATTGGGGTCAGCACAGATCT
\end{tabular}

TNF- $\alpha$, tumor necrosis factor- $\alpha$; IL- $1 \beta$, interleukin- $1 \beta$; IFN- $\gamma$, interferon- $\gamma$.

(Sigma-Aldrich, St. Louis, MO, USA), dissolved in 90:10 propylene glycol:ethanol at $0.5 \mu \mathrm{g} / \mathrm{kg}$ body weight, while the vehicle subgroups received the solvent only. Paricalcitol or vehicle was administered through i.p. injection 30 min prior to exposure to hyperoxia, and on every other day $(1,3,5 \ldots 21)$ afterwards.

Histology. Lungs were collected and sectioned at a thickness of $4 \mu \mathrm{m}$. The slides were stained with H\&E (Beyotime Institute of Biotechnology, Haimen, China) at room temperature. Radial alveolar counts (RAC) were measured by drawing a perpendicular line from the center of the most peripheral bronchiole to the pleura or the nearest interlobular septum and counting the number of alveoli transected by this line. Every section was evaluated by two pathologists who were blind to the study design.

Masson-trichrome staining was performed using a commercial kit (Beyotime Institute of Biotechnology) according to the instructions. ECM deposition was analyzed by Image Pro Plus 6.0 (Media Cybernetics, Rockville, MD, USA).

Wet/dry ratio of the lung weight. The right upper lung lobes were excised and weighed to determine the wet lung weight. They were dried in an oven at $80^{\circ} \mathrm{C}$ for $48 \mathrm{~h}$ until the weights stopped to change. They were then weighed again for the dry weight. Wet/dry ratio of the lung weight was calculated accordingly.

Bronchoalveolar lavage fluid (BALF) collection. After anesthesia by intraperitoneal injection with a cocktail of xylazine (Rompun 2\%; Bayer AG, Leverkusen, Germany) and ketamine (Ketavest; $100 \mathrm{mg} / \mathrm{ml}$; Pfizer, Inc., New York, NY, USA), the trachea was exposed and the lungs were lavaged three times with $0.2 \mathrm{ml}$ sterile saline/wash. The fluids were collected and stored at $4^{\circ} \mathrm{C}$. The BCA method was used to test the protein concentrations in the fluids.

Real-time PCR. Total RNA was isolated from the lung tissues using TRIzol reagent (Invitrogen, Carlsbad, CA, USA). First-strand cDNAs were synthesized with a PrimeScript RT reagent kit (Takara Biotechnology Co., Ltd., Dalian, China). Real-time PCR was performed in a $20-\mu 1$ mixture using a SYBR-Green PCR reagent kit (Clontech Laboratories, Inc., Mountainview, CA, USA) and a Bio-Rad IQ5 real-time system. The $2^{-\Delta \Delta \mathrm{Cq}}$ formula was used to quantify the relative expression of mRNA. $\beta_{2}$ microglobulin $(\mathrm{B} 2 \mathrm{M})$ was used as an internal control. Sequences of the PCR primers are provided in Table I.

Terminal deoxynucleotidyltransferase-mediated dUTP nick-end labelling (TUNEL) staining. TUNEL staining was performed using an In Situ Cell Death Detection kit, TMR red (Roche Diagnostics, Indianapolis, IN, USA) according to the manufacturer's instructions. The number of TUNEL-positive cells/field was recorded and compared statistically.

Western blot analysis. Total proteins were harvested from the lung tissue. Equal amounts of proteins (50 $\mu \mathrm{g} / \mathrm{lane})$ were separated by $10 \%$ polyacrylamide gel electrophoresis, and the proteins were transferred electrophoretically onto polyvinylidene difluoride membranes (EMD Millipore, Billerica, MA, USA). The membranes were then incubated with primary antibodies. The primary antibodies and their concentrations were as follows: anti-TLR4 (1:1,000 dilution; ab30667), anti-caspase-3 (1:1,000 dilution; ab52293) and anti- $\beta$-actin (1:5,000 dilution; ab8226) (all from Abcam). After being washed with TBST 3 times, the membranes were then incubated at room temperature for $1 \mathrm{~h}$ with horseradish peroxidase-conjugated secondary antibodies (goat anti-rabbit and anti-mouse IgG-HRP; Cat. nos. sc-2004 and sc-2005, respectively; 1:2,000 dilution; Santa Cruz Biotechnology, Inc., Santa Cruz, CA, USA).

Statistical analyses. All continuous data are presented as mean \pm standard deviation (SD). Statistical comparison of continuous variables between groups was performed using the Student's t-test or one-way ANOVA (followed by Games-Howell test) with GraphPad Prism software 6.0 (GraphPad Software Inc., La Jolla, CA, USA) and SPSS 17.0 (SPSS Inc., Chicago, IL, USA). Rank data were analyzed using Wilcoxon rank test. $\mathrm{P}<0.05$ was considered to indicate a statistically significant result.

\section{Results}

Vitamin $D$ attenuates developmental retardation caused by hyperoxia and promotes the survival rate. In rats treated with hyperoxia, developmental retardation was observed from day 3 , as represented by a decreased body weight compared to the control groups. Vitamin D significantly 

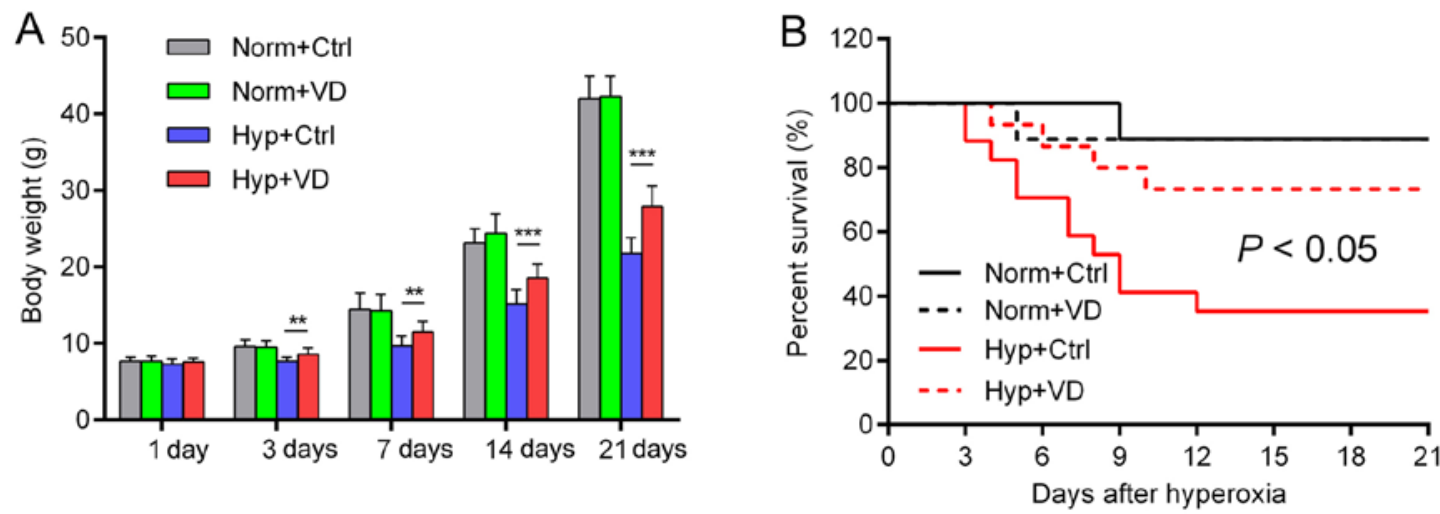

Figure 1. General status of research animal groups. (A) The average body weight of the four rat groups. (B) Rates of survival in the four groups. $n=9$ in the normoxia group, $\mathrm{n}=15-17$ in the hyperoxia group. Norm + Ctrl, normoxia and vehicle; Norm+VD, normoxia and paricalcitol; Hyp+Ctrl, hyperoxia and vehicle; Hyp+VD, hyperoxia and paricalcitol. $\mathrm{P}<0.05$ refers to the difference between the survival rates of the Hyp+Ctrl and Hyp+VD group. ${ }^{* *} \mathrm{P}<0.01$ and ${ }^{* * * *} \mathrm{P}<0.001$.
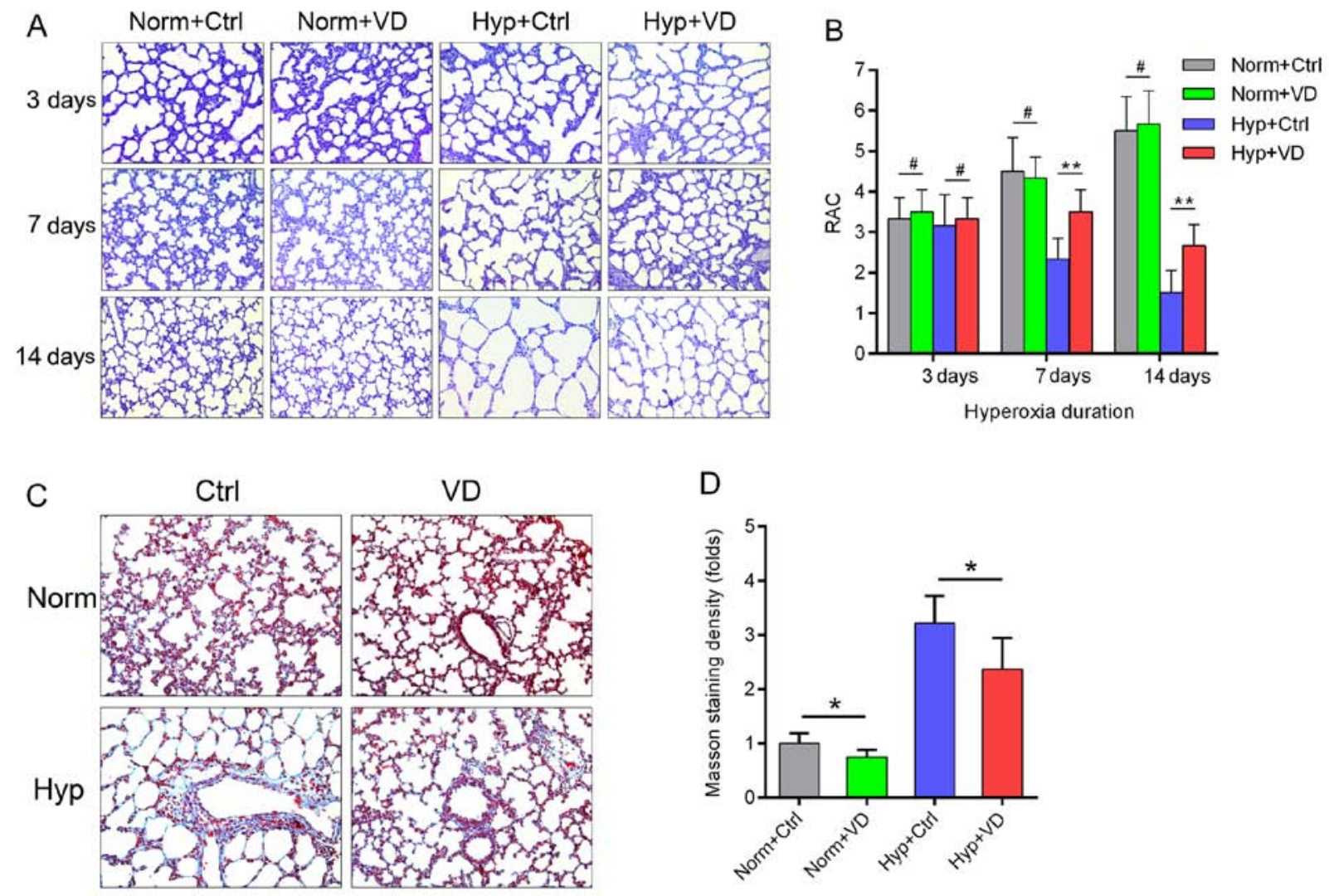

Figure 2. Morphological changes of the lungs. (A) H\&E staining of the lungs 3, 7 and 14 days after exposure to hyperoxia (n=6 in each group). Magnification, $\mathrm{x} 200$. (B) Radial alveolar counts (RAC) in the lungs 3, 7 and 14 days after exposure of hyperoxia ( $\mathrm{n}=6$ in each group). ${ }^{*} \mathrm{P}>0.05$ and ${ }^{* *} \mathrm{P}<0.01$. (C) Masson staining of the lungs ( $\mathrm{n}=6$ in each group). Magnification, $\mathrm{x} 200$. (D) Fold-change in Masson staining density compared to the Norm+Ctrl group ( $\mathrm{n}=6$ in each group); ${ }^{*} \mathrm{P}<0.05$. Norm+Ctrl, normoxia and vehicle; Norm+VD, normoxia and paricalcitol; Hyp+Ctrl, hyperoxia and vehicle; Hyp+VD, hyperoxia and paricalcitol.

attenuated this retardation, but did not influence the body weights of the normoxia groups (Fig. 1A). Only $35.3 \%$ of the rats survived hyperoxia, while vitamin $\mathrm{D}$ increased the rate to $73.3 \%$ (Fig. 1B). Vitamin D reduced the developmental retardation and mortality caused by hyperoxia $(n=9$ in the normoxia group, $\mathrm{n}=15-17$ in the hyperoxia group).

Vitamin D preserves lung structure and decreases extracellular matrix deposition induced by hyperoxia. We investigated lung structures microscopically. Hyperoxia led to aberrance, infiltration of neutrophils and destruction of normal alveolar walls. Decrease in the numbers of alveoli and secondary septa, irregular alveolar shape and thickening of the alveolar wall were also observed. Compared to the control groups, vitamin D-treated lungs presented with more intact structures and less inflammation (Fig. 2A). RAC, which represent the status of alveolarization, started to differ 7 days after hyperoxia (Fig. 2B). Masson staining was performed in rats after 7 days of hyperoxia. Hyperoxia induced extracellular matrix deposition in the alveolar wall, while vitamin D attenuated 

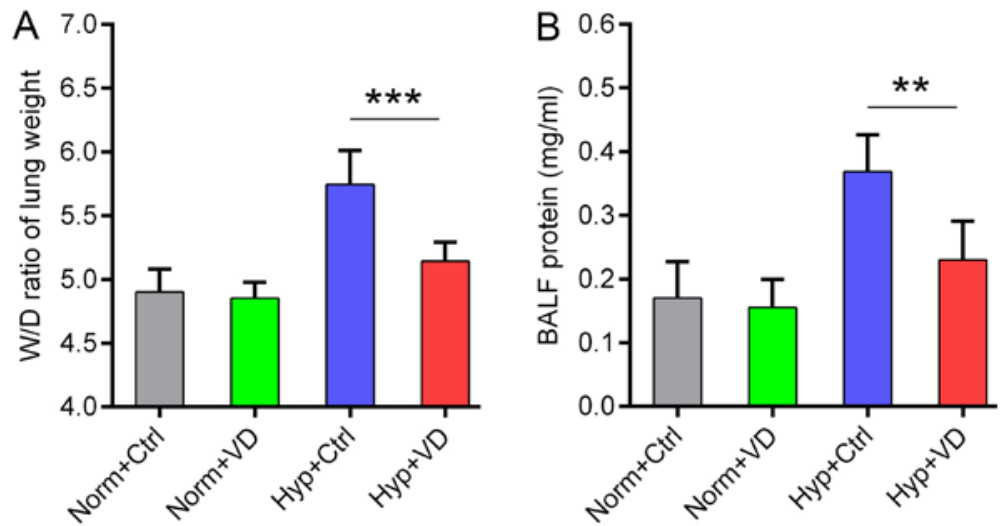

Figure 3. Extent of lung edema and barrier functions. (A) The wet/dry (W/D) ratio of lung weights ( $\mathrm{n}=6$ in each group). (B) The protein concentration in the bronchoalveolar lavage fluid (BALF) ( $\mathrm{n}=6$ in each group). Norm+Ctrl, normoxia and vehicle; Norm+VD, normoxia and paricalcitol; Hyp+Ctrl, hyperoxia and vehicle; Hyp+VD, hyperoxia and paricalcitol. ${ }^{* *} \mathrm{P}<0.01$ and ${ }^{* * *} \mathrm{P}<0.001$.

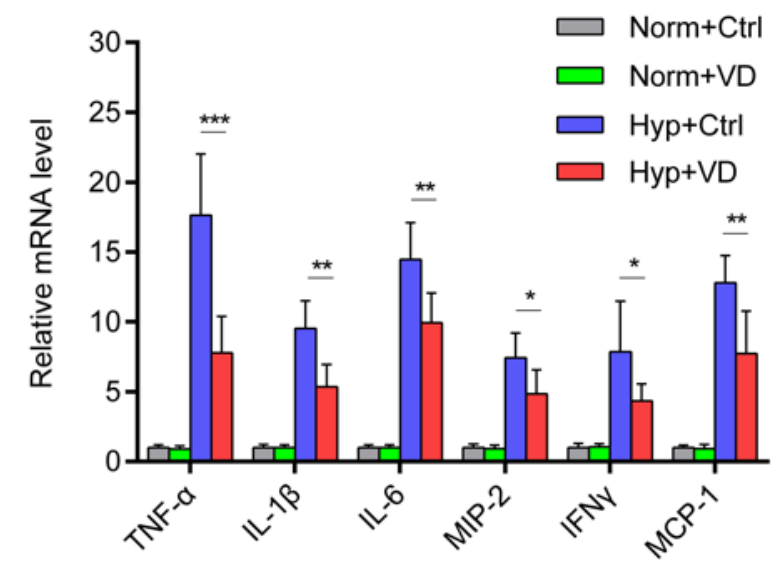

Figure 4. Expression of inflammatory cytokines and chemokines ( $n=6-8$ in each group). Norm+Ctrl, normoxia and vehicle; Norm+VD, normoxia and paricalcitol; Hyp+Ctrl, hyperoxia and vehicle; Hyp+VD, hyperoxia and paricalcitol. ${ }^{*} \mathrm{P}<0.05,{ }^{* *} \mathrm{P}<0.01$ and ${ }^{* * * *} \mathrm{P}<0.001$.

this change. This attenuating effect was also observed in the normoxia groups (Fig. 2C and D) ( $\mathrm{n}=6$ in each group).

Vitamin D relieves lung edema, maintains barrier function and inhibits inflammation in hyperoxia-treated rats. We recorded the wet/dry ratio of the lung weight in all the groups, which is a good indicator of lung edema. Hyperoxia increased this ratio, indicating more water leakage into the alveolar space. Vitamin D treatment significantly attenuated lung edema (Fig. 3A). Similar change was also observed in the BALF protein content, showing the protective effect of vitamin $\mathrm{D}$ on the epithelial cell barrier function (Fig. 3B) $(n=6$ in each group). Then we measured the expression of inflammatory mRNAs in the lung tissue. Hyperoxia stimulated the expression of inflammatory cytokines and chemokines, while vitamin D significantly inhibited the stimulation (Fig. 4) ( $n=6-8$ in each group).

Vitamin D decreases alveolar cell apoptosis by downregulating TLR4. We performed TUNEL staining to investigate apoptosis in the alveolar cells 7 days after exposure to hyperoxia. In the normoxia groups, TUNEL-positive cells were scarce. Hyperoxia led to a marked increase in the number of TUNEL-positive cells, while vitamin $\mathrm{D}$ was protective against hyperoxia-induced apoptosis (Fig. 5A). The apoptotic index was calculated as the number of apoptotic cells/field, which was significantly increased by hyperoxia and ameliorated by vitamin D (Fig. 5B). Western blotting showed activation of TLR4 and a subsequent increase in the expression of caspase-3, a downstream apoptotic protein in the hyperoxic group. Vitamin D treatment suppressed TLR4 expression and subsequently the expression of cleaved caspase-3 (Fig. 5C and D) ( $n=6$ in each group).

\section{Discussion}

This study demonstrated that vitamin D attenuates hyperoxia-induced lung injury in neonatal rats, by protecting the integrity of the lung structure, decreasing ECM deposition and inhibiting inflammation. The mechanism may lie in the anti-apoptotic effect of vitamin D by downregulating TLR4.

Hyperoxia-induced lung injury has been a focus of recent research, for it best simulates BPD, a disease that severely impairs the lung function in preterm births. The number of macrophages, the surface volume of gas exchange and the volume of lung parenchyma are decreased, while the area of atelectasis is increased (17). Hyperoxia induces the production of ROS, which subsequently activates several downstream pathways, represented by the MAPK and NF- $\mathrm{KB}$ cascades $(4,18)$. Therefore it causes the release and accumulation of inflammatory mediators in the alveolar space (19). Moreover, hyperoxia may destruct both the endothelial and the epithelial barriers by downregulating tight junction proteins $(20,21)$. We also observed deposition of extracellular matrix and retarded lung development, as reported in our preview study (22).

Through the production of ROS, hyperoxia regulates the expression of TLRs, and thereby activates NF- $\kappa B$-related expression of inflammatory cytokines (4). TLRs are important components of the innate immunity, which can identify all types of microbial structures. In the lung, they protect the host from environmental oxidants and invading microbes (23). As the receptor of LPS and a key component in the NF- $\kappa$ B pathway, TLR4 is essential for survival and lung integrity, by sensing microbial components, exogenous oxidants and 
A
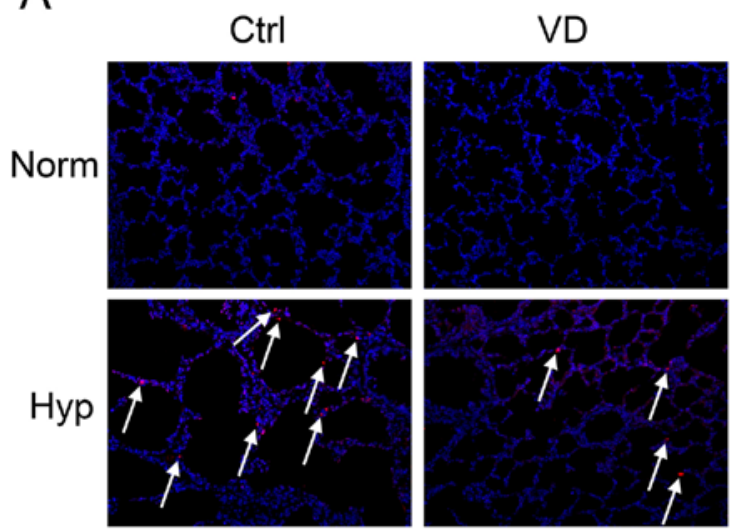

C

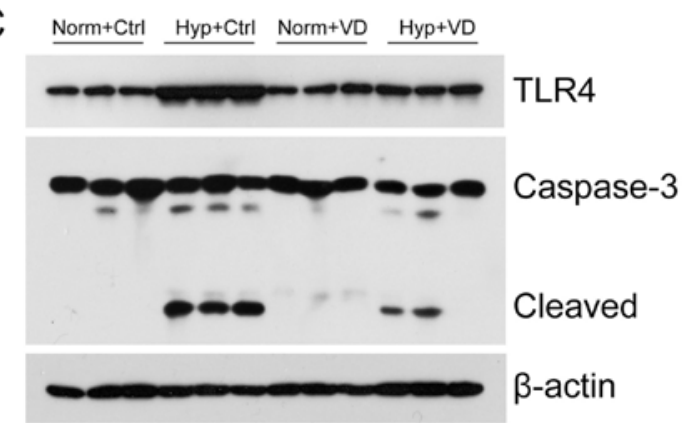

B

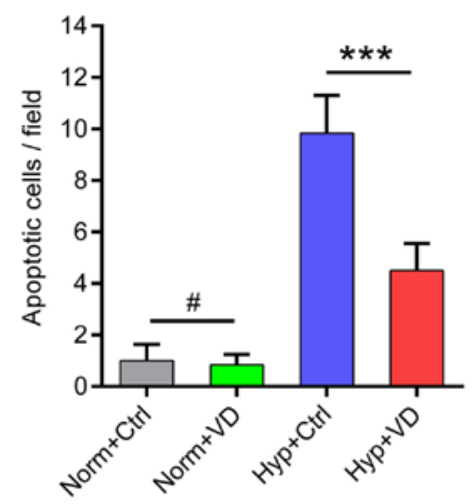

$\mathrm{D}$

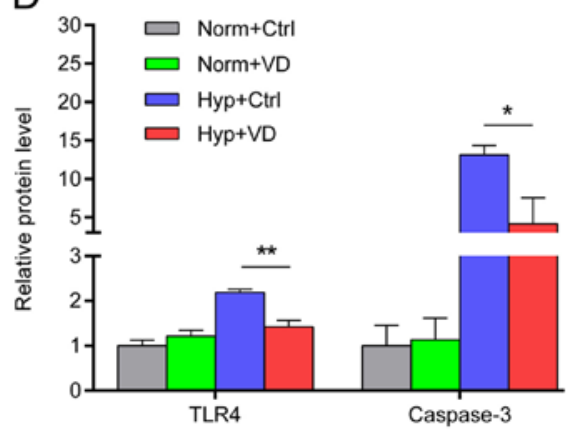

Figure 5. Alveolar epithelial cell apoptosis and the expression of Toll-like receptor 4 (TLR4). (A) TUNEL staining of the lung tissues. Arrows refer to the apoptotic cells ( $\mathrm{n}=6$ in each group). Magnification, $\mathrm{x} 200$. (B) Apoptotic index, calculated as the number of apoptotic cells/field ( $\mathrm{n}=6$ in each group). ${ }^{\#} \mathrm{P}>0.05$ and ${ }^{* * * *} \mathrm{P}<0.001$. (C) Western blotting of TLR4, caspase- 3 and its cleaved form ( $\mathrm{n}=6$ in each group). (D) Relative protein quantification of TLR4 and caspase- 3 ( $\mathrm{n}=6$ in each group). ${ }^{*} \mathrm{P}<0.05$ and ${ }^{* *} \mathrm{P}<0.01$. Norm $+\mathrm{Ctrl}$, normoxia and vehicle; Norm+VD, normoxia and paricalcitol; Hyp+Ctrl, hyperoxia and vehicle; Hyp+VD, hyperoxia and paricalcitol.

modulating inflammatory and apoptotic responses (24). Mutation or deletion of TLR4 has an anti-inflammatory effect on endotoxin-induced inflammation and ischemic-reperfusion injury (19). Activation of TLR4 is also responsible for neonatal necrotizing enterocolitis-associated lung injury, which has a similar but more severe pathological process compared to other forms of premature-associated lung injury (25).

Hyperoxia has been found to elevate the expression of TLR4 in A549 cells (5), and in this study, in neonatal rat lungs. This over-activation of TLR4 led to increased release of inflammatory mediators including tumor necrosis factor- $\alpha$ (TNF- $\alpha)$, interleukin-1 $\beta$ (IL-1 $\beta$ ), IL-6, MIP-2, interferon- $\gamma($ IFN- $\gamma)$ and MCP-1. Over-activation of TLR4 has been reported to promote neutrophil recruitment and microvascular and alveolar epithelial injury in a dose-dependent manner (26). Apoptosis was induced subsequently, as confirmed by increases in the number of TUNEL-positive cells and the expression of caspase-3.

Vitamin D largely preserved normal lung structure in the hyperoxia-induced lung injury. The aberrance, mal-development of the alveoli and the deposition of extracellular matrix were attenuated by vitamin D. We observed less infiltration of neutrophils and reduction in the release of pro-inflammatory cytokines, indicating an anti-inflammatory effect of vitamin D on hyperoxia-induced lung injury. This effect has also been observed in models of inflammatory bowel diseases and ischemic-reperfusion injury $(8,9)$. Our previous study found that vitamin $\mathrm{D}$ receptor-knockout mice showed a more intense inflammatory response to LPS and vitamin D treatment decreased the secretion of pro-inflammatory cytokines in LPS-induced lung injury (11). The anti-apoptotic role of vitamin D was also demonstrated in pulmonary tissue (10). Agonists of vitamin D receptor relieved cardiomyocyte apoptosis in a model of myocardial ischemic-reperfusion (27). Vitamin D ameliorated intestinal epithelial apoptosis in 2,4,6-trinitrobenzene sulphonic acid-induced colitis by inhibiting the expression of caspase-3 and p53 (28). Such an effect was also observed in pulmonary epithelial cells (11).

Many studies have indicated that these anti-inflammatory and anti-apoptotic effects of vitamin D function through the TLR4 pathway. Vitamin D has been demonstrated to downregulate TLR4 in liver cells, human monocytes THP-1, antigen-presenting cells and keratinocytes, resulting in a lower inflammatory response and reduced apoptosis (13-15,29). While hyperoxia upregulated the expression of TLR4, vitamin $\mathrm{D}$ treatment significantly attenuated this increase, and thereby exerted its anti-inflammatory and anti-apoptotic functions.

In conclusion, hyperoxia may destruct lung structure, retard lung development and cause deposition of extracellular matrix. The release of pro-inflammatory cytokines and the increase in pulmonary epithelial cell apoptosis may be caused by activation of TLR4. Vitamin D administration can antagonize the activation of TLR4 and therefore alleviate inflammation, reduce apoptosis and preserve lung structure. Since hyperoxia-induced lung injury is a typical model of 
BPD, vitamin D may be of curative or preventive value to patients with BPD.

\section{Acknowledgements}

This study was supported by the National Natural Science Foundation of China (nos. 81571479 and 81471489).

\section{References}

1. Kair LR, Leonard DT and Anderson JM: Bronchopulmonary dysplasia. Pediatr Rev 33: 255-263, 2012.

2. Balany $J$ and Bhandari V: Understanding the impact of infection, inflammation and their persistence in the pathogenesis of bronchopulmonary dysplasia. Front Med (Lausanne) 2: 90, 2015.

3. Silva DM, Nardiello C, Pozarska A and Morty RE: Recent advances in the mechanisms of lung alveolarization and the pathogenesis of bronchopulmonary dysplasia. Am J Physiol Lung Cell Mol Physiol 309: L1239-L1272, 2015.

4. Chen Y, Li Q, Liu Y, Shu L, Wang N, Wu Y, Sun X and Wang L: Attenuation of hyperoxia-induced lung injury in neonatal rats by 1 $\alpha$, 25-Dihydroxyvitamin $\mathrm{D}_{3}$. Exp Lung Res 41: 344-352, 2015.

5. Huang D, Fang F and Xu F: Hyperoxia induces inflammation and regulates cytokine production in alveolar epithelium through TLR2/4-NF- $\mathrm{BB}$-dependent mechanism. Eur Rev Med Pharmacol Sci 20: 1399-1410, 2016.

6. Zhao Y, Zhao Y, Zhang M, Zhao J, Ma X, Huang T, Pang H, Li J and Song J: Inhibition of TLR4 signalling-induced inflammation attenuates secondary injury after diffuse axonal injury in rats. Mediators Inflamm 2016: 4706915, 2016.

7. Perros F, Lambrecht BN and Hammad H: TLR4 signaling in pulmonary stromal cells is critical for inflammation and immunity in the airways. Respir Res 12: 125, 2011.

8. Liu TJ, Shi YY, Du J, Ge X, Teng X, Liu L, Wang EB and Zhao Q: Vitamin D treatment attenuates 2, 4, 6-trinitrobenzene sulphonic acid (TNBS)-induced colitis but not oxazolone-induced colitis. Sci Rep 6: 32889, 2016

9. Lee JW, Kim SC, Ko YS, Lee HY, Cho E, Kim MG, Jo SK Cho WY and Kim HK: Renoprotective effect of paricalcitol via a modulation of the TLR4-NF- $\kappa$ B pathway in ischemia/ reperfusion-induced acute kidney injury. Biochem Biophys Res Commun 444: 121-127, 2014

10. Kose M, Bastug O, Sonmez MF, Per S, Ozdemir A, Kaymak E, Yahşi $\mathrm{H}$ and Ozturk MA: Protective effect of vitamin D against hyperoxia-induced lung injury in neonatal rats. Pediatr Pulmonol 52: 69-76, 2017.

11. Shi YY, Liu TJ, Fu JH, Xu W, Wu LL, Hou AN and Xue XD: Vitamin D/VDR signaling attenuates lipopolysaccharideinduced acute lung injury by maintaining the integrity of the pulmonary epithelial barrier. Mol Med Rep 13: 1186-1194, 2016.

12. Kong J, Zhu X, Shi Y, Liu T, Chen Y, Bhan I, Zhao Q, Thadhani $\mathrm{R}$ and Li YC: VDR attenuates acute lung injury by blocking Ang-2-Tie-2 pathway and renin-angiotensin system. Mol Endocrinol 27: 2116-2125, 2013.

13. Verma R, Jung JH and Kim JY: 1,25-Dihydroxyvitamin $\mathrm{D}_{3}$ upregulates TLR10 while downregulating TLR2, 4 and 5 in human monocyte THP-1. J Steroid Biochem Mol Biol 141: 1-6, 2014.

14. Wang H, Zhang Q, Chai Y, Liu Y, Li F, Wang B, Zhu C, Cui J, $\mathrm{Qu} \mathrm{H}$ and $\mathrm{Zhu}$ Ma: $1,25(\mathrm{OH})_{2} \mathrm{D}_{3}$ downregulates the Toll-like receptor 4-mediated inflammatory pahway and ameliorates liver injury in diabetic rats. J Endocrinol Invest 38: 1083-1091, 2015.

15. Gambhir V, Kim J, Siddiqui S, Taylor M, Byford V, Petrof EO, Jones $\mathrm{G}$ and Basta S: Influence of 1,25-dihydroxy vitamin D3 on TLR4-induced activation of antigen presenting cells is dependent on the order of receptor engagement. Immunobiology 216 : 988-996, 2011.
16. Hou AN, Fu JH, Yang HP, Zhu YT, Pan YQ, Xu SY and Xue XD: Hyperoxia stimulates the transdifferentiation of type II alveolar epithelial cells in newborn rats. Am J Physiol Lung Cell Mol Physiol 308: L861-L872, 2015.

17. Reis RB, Nagato AC, Nardeli CR, Matias IC, Lima WG and Bezerra FS: Alternations in the pulmonary histoarchitecture of neonatal mice exposed to hyperoxia. J Pediatr (Rio J) 89: 300-306, 2013

18. Porzionato A, Sfriso MM, Mazzatenta A, Macchi V, De Caro R and Di Giulio C: Effects of hyperoxic exposure on signal transduction pathways in the lung. Respir Physiol Neurobiol 209: 106-114, 2015.

19. Molteni M, Gemma S and Rossetti C: The role of TLR4 in infectious and non-infectious inflammation. Mediators Inflamm 2016: 6978936, 2016.

20. You K, Xu XW, Fu JH, Xu SY, Yue XH, Yu ZL and Xue XD: Hyperoxia disrupts pulmonary epithelial barrier in newborn rats via the deterioration of occludin and ZO-1. Respir Res 13: 36, 2012.

21. Li C, Fu JH, Liu HY, Yang HP, Yao L, You K and Xue XD: Hyperoxia arrests pulmonary development in newborn rats via disruption of endothelial tight junctions and downregulation of CX40. Mol Med Rep 10: 61-67, 2014.

22. Yang HP, Fu JH, Xue XD, Yao L, Qiao L, Hou AN, Jin LL and Xing YJ: Epithelial-mesenchymal transitions in bronchopulmonary dysplasia of newborn rats. Pediatr Pulmonol 49: 1112-1123, 2014.

23. Qureshi ST, Zhang X, Aberg E, Bousette N, Giaid A, Shan P, Medzhitov RM and Lee PJ: Inducible activation of TLR4 confers resistance to hyperoxia-induced pulmonary apoptosis. J Immunol 176: 4950-4958, 2006.

24. Zhang X, Shan P, Qureshi S, Homer R, Medzhitov R, Noble PW and Lee PJ: Cutting edge: TLR4 deficiency confers susceptibility to lethal oxidant lung injury. J Immunol 175: 4834-4838, 2005.

25. Jia H, Sodhi CP, Yamaguchi Y, Lu P, Martin LY, Good M, Zhou Q, Sung J, Fulton WB, Nino DF, et al: Pulmonary epithelial TLR4 activation leads to lung injury in neonatal necrotizing enterocolitis. J Immunol 197: 859-871, 2016.

26. Togbe D, Schnyder-Candrian S, Schnyder B, Couillin I, Maillet I, Bihl F, Malo D, Ryffel B and Quesniaux VF: TLR4 gene dosage contributes to endotoxin-induced acute respiratory inflammation. J Leukoc Biol 80: 451-457, 2006.

27. Yao T, Ying X, Zhao Y, Yuan A, He Q, Tong H, Ding S, Liu J, Peng X, Gao E, et al: Vitamin D receptor activation protects against myocardial reperfusion injury through inhibition of apoptosis and modulation of autophagy. Antioxid Redox Signal 22: 633-650, 2015.

28. Zhu T, Liu TJ, Shi YY and Zhao Q: Vitamin D/VDR signaling pathway ameliorates 2,4,6-trinitrobenzene sulphonic acid-induced colitis by inhibiting intestinal epithelial apoptosis. Int J Mol Med 35: 1213-1218, 2015.

29. Jeong MS, Kim JY, Lee HI and Seo SJ: Calcitriol may downregulate mRNA over-expression of Toll-like receptor- 2 and 4 , $L L-37$ and proinflammatory cytokines in cultured human keratocytes. Ann Dermatol 26: 296-302, 2014. 